INPLASY

PROTOCOL

To cite: Mao et al. Pediatric Tui $\mathrm{Na}$ for cough in children: $\mathrm{A}$ protocol for a systematic review and meta-analysis. Inplasy protocol 202220076. doi:

10.37766/inplasy2022.2.0076

Received: 18 February 2022

Published: 19 February 2022

Corresponding author:

Xun Li

tina000341@163.com

Author Affiliation:

Beijing University of Chinese Medicine.

Support: None.

Review Stage at time of this submission: Data extraction.

Conflicts of interest: None declared.

\section{Pediatric Tui Na for cough in children: A protocol for a systematic review and meta-analysis}

\author{
Mao, H Wei, YH²; Su, HM33; Li, X4.
}

Review question / Objective: The aim of this systematic review is to evaluate the effectiveness and safety of pediatric Tui $\mathrm{Na}$ in the treatment of cough in children under seven years of age.

Condition being studied: Cough is essentially a protective reflex of respiratory tract to various stimuli, typically in order to clear the lung airways of fluids, mucus, or other material. Cough not only has a negative impact on children's daily activities and sleep, but is associated with parental stress and worries. Pediatric Tui $\mathrm{Na}$, a therapeutic massage based on the Chinese traditional theory of Yin and Yang, Qi and blood, acupoints and meridians, enjoys a long history and has been widely applied to the treatment of common diseases like fever, diarrhea, cough and asthma. This study aims to evaluate the effectiveness and safety of pediatric Tui $\mathrm{Na}$ in the treatment of cough in children.

INPLASY registration number: This protocol was registered with the International Platform of Registered Systematic Review and Meta-Analysis Protocols (INPLASY) on 19 February 2022 and was last updated on 19 February 2022 (registration number INPLASY202220076).

\section{INTRODUCTION}

Review question / Objective: The aim of this systematic review is to evaluate the effectiveness and safety of pediatric Tui $\mathrm{Na}$ in the treatment of cough in children under seven years of age.
Condition being studied: Cough is essentially a protective reflex of respiratory tract to various stimuli, typically in order to clear the lung airways of fluids, mucus, or other material. Cough not only has a negative impact on children's daily activities and sleep, but is associated with 
parental stress and worries. Pediatric Tui $\mathrm{Na}$, a therapeutic massage based on the Chinese traditional theory of Yin and Yang, Qi and blood, acupoints and meridians, enjoys a long history and has been widely applied to the treatment of common diseases like fever, diarrhea, cough and asthma. This study aims to evaluate the effectiveness and safety of pediatric Tui $\mathrm{Na}$ in the treatment of cough in children.

\section{METHODS}

Search strategy: Terms (taking PubMed as an example): randomized controlled trial; controlled clinical trial; randomized; placebo; drug therapy; randomly; trial; groups; tuina; tui na; massage; chiropractic; acupressure; musculoskeletal manipulation; spine manipulation; infant; child; kid; toddler; baby; cough; croup; tussis; pertussis; begma. We also searched the following databases: (1) Cochrane Library. (2) Embase. (3) CNKI. (4) Wanfang Data. (5) VIP Database. (6) SinoMed. (7) ClinicalTrials.gov. (8) WHO ICTRP.

Participant or population: Children with cough under seven years of age. Cough is related to various respiratory diseases, but we didn't impose any restriction on disease types when searching for potential trials. As clinical practice guidelines on pediatric Tui $\mathrm{Na}$ for cough were not available, we referred to a guide to massage in the treatment of acute diarrhea in children, which explained that children aged 0-6 were the major applicable objects of the handbook. Textbooks on acupuncture and massage also pointed out that pediatric Tui $\mathrm{Na}$ is suitable for young children, especially infants; for older ones, the manipulation should be adapted, often combined with techniques for adults. In addition, we consulted pediatricians to confirm the consistence with clinical practice. Taking all these into consideration, we set the age limit at $0-6$ years.

Intervention: Any form of pediatric Tui $\mathrm{Na}$ including manipulation on any part of the body, acupressure, abdominal massage and spine manipulation.
Comparator: Trials comparing pediatric Tui $\mathrm{Na}$ with no intervention, placebo drug, or conventional therapy. Also included were comparisons of pediatric Tui $\mathrm{Na}$ combined with conventional treatment versus the same conventional treatment alone in the management of pediatric cough. Studies in which pediatric Tui Na was combined with other TCM therapies were excluded.

Study designs to be included: Randomized controlled trials.

Eligibility criteria: RCTs reported as full text or unpublished. Restrictions on language, time frame or country were not applied during study selection.

Information sources: Electronic databases including PubMed, Cochrane Library, EMBASE, CNKI, Wanfang Data, VIP Database and SinoMed were searched for the systematic review. We also conducted a search of ClinicalTrials.gov and the World Health Organization trials portal (WHO ICTRP) for ongoing studies.

Main outcome(s): Cough related status (such as cough frequency, severity, and duration).

Additional outcome(s): (1) Use of Western medicines. (2) Quality of life. (3) Medical costs. (4) Recurrence rate. (5) Adverse events.

Data management: Two review authors will independently scan and screen the studies with the software of NoteExpress. We will extract the data of included trials with a structured data extraction form, including the study design, sample size, participants' baseline characteristics, intervention, control and outcome measures. Disagreements will be resolved through discussion or by consultation with a third review author.

Quality assessment / Risk of bias analysis: Two review authors will independently assess the risk of bias for the included studies using the Cochrane Collaboration Bias Risk Assessment tool. The evaluation content will include the following domains: 
(1) Random sequence generation. (2) Allocation concealment. (3) Blinding of participants and personnel. (4) Blinding of outcome assessment. (5) Incomplete outcome data. (6) Selective outcome reporting. (7) Other bias. We will also use the five GRADE considerations (risk of bias, inconsistency, indirectness, imprecision and other considerations) to assess the quality of evidence for the main outcomes.

Strategy of data synthesis: We will use Review Manager Software 5.4 to conduct meta-analyses. Risk ratios (RRs) will be applied for dichotomous data and mean differences (MDs) for continuous data, both with $95 \%$ confidence intervals (Cls). As disparity in Tui $\mathrm{Na}$ forms and medications between different studies may exist, a random-effect model will be applied in meta-analyses. The 12 statistic will be used to assess heterogeneity. Values of $30 \%$ or higher suggest heterogeneity, and causes for it will be explored by subgroup analyses. If 12 is lower than $30 \%$, we will then conduct meta-analyses with a fixedeffect model for sensitivity analyses.

Subgroup analysis: We will conduct a priori subgroup analyses according to disease diagnosis or medication.

Sensitivity analysis: We will conduct sensitivity analyses to assess the impact of risk of bias on main outcomes by including or excluding trials judged as "high risk of bias". If there are different studies regarding disease status, sample size, intervention, comparator or outcomes, we will also carry out sensitivity analyses to test the robustness of the meta-analysis result.

Language: No language restriction was imposed on the search.

Country(ies) involved: China.

Keywords: Pediatric Tui Na; cough; randomized controlled trial; systematic review; meta-analysis.

Contributions of each author:

Author 1 - Hui Mao.
Author 2 - YueHui Wei. Author 3 - Huimin Su. Author 4 - Xun Li. 